\title{
Telavancin for the treatment of nosocomial pneumonia caused by methicillin-resistant Staphylococcus aureus (MRSA)
}

This article was published in the following Dove Press journal:

Therapeutics and Clinical Risk Management

15 March 2012

Number of times this article has been viewed

\author{
Candace $Y$ Hooper \\ Winter J Smith \\ Department of Pharmacy, Clinical \\ and Administrative Sciences, \\ University of Oklahoma Health \\ Sciences Center College of Pharmacy, \\ Oklahoma City, OK, USA
}

\begin{abstract}
Telavancin is a bactericidal lipoglycopeptide antibiotic that is structurally related to vancomycin. It demonstrates in vitro activity against a variety of Gram-positive pathogens including, but not limited to, methicillin-resistant Staphylococcus aureus (MRSA). Telavancin is currently FDA-approved for the treatment of complicated skin and skin-structure infections. Recently, two randomized clinical trials demonstrated the efficacy and safety of telavancin compared to vancomycin for the treatment of nosocomial pneumonia. Overall, telavancin has a favorable safety profile. However, mild gastrointestinal disturbances and reversible increases in serum creatinine were observed in clinical studies. Additional clinical studies are needed to evaluate telavancin's efficacy and safety in comparison to other antistaphylococcal agents for the treatment of infections such as bacteremia and endocarditis.
\end{abstract}

Keywords: telavancin, MRSA, hospital-acquired pneumonia, health care-associated pneumonia, ventilator-associated pneumonia, nosocomial pneumonia

\section{Introduction}

Nosocomial pneumonia describes hospital-acquired pneumonia (HAP), health careassociated pneumonia (HCAP), or ventilator-associated pneumonia (VAP); all of which are delineated primarily based on the time of onset and etiology of infection. HAP, pneumonia that occurs greater than 48 hours after admission, is the second most common nosocomial infection in the United States (US). ${ }^{1}$ HCAP refers to pneumonia acquired outside of the hospital by patients with certain risk factors for infection by pathogens of a nosocomial origin. These risk factors include hospitalization in an acute care facility for two or more days in the previous 90 days; residence in a nursing home or long-term care facility; previous intravenous antibiotic therapy, chemotherapy, or wound care within the past 30 days of the current infection; or hemodialysis in the hospital or clinic. In contrast, VAP is pneumonia that occurs at least 48 hours after endotracheal intubation. ${ }^{1}$ Both HAP and VAP remain contributors to poor patient outcomes despite advances in antibiotic therapy and the implementation of preventative measures. ${ }^{1}$ HAP has an average incidence of 5 to 10 cases per 1000 hospitalized patients, and an estimated attributable mortality of up to $50 \% .^{1,2}$ A HAP diagnosis increases the length of hospitalization by an average of 7 to 9 days per patient, costing a reported excess of US $\$ 40,000$ per patient. ${ }^{3,4}$

HAP can be caused by a variety of organisms including aerobic Gram-negative bacilli and Gram-positive cocci, particularly methicillin-resistant Staphylococcus aureus (MRSA). ${ }^{1,2}$ HAP caused by $S$. aureus has become a major epidemiological focal point, considering the rapid emergence of resistant strains with limited treatment 
options and their impact on mortality. In an analysis of a large US inpatient database, $S$. aureus was found to be the only pathogen among those causing nosocomial pneumonia to be associated with a significant increase in mortality. ${ }^{2}$ The same study showed that patients infected with MRSA were more likely to receive inappropriate antibiotic therapy. ${ }^{2}$ Inadequate therapy is associated with both an increase in pneumonia treatment failure rates and mortality. ${ }^{1}$

Risk factors for HAP have primarily been extrapolated from patients diagnosed with VAP. These risk factors are categorized as modifiable and nonmodifiable (Table 1). ${ }^{1,5,6}$ Identifying and addressing modifiable risk factors could potentially aid in HAP management. These risk factors may also be important to consider when selecting antimicrobial therapy. The time of onset of clinical signs of pneumonia serves as a helpful indicator in determining likely pathogens and potential patient outcomes associated with HAP. Earlyonset HAP (occurring within the first 4 days of hospital admission) is more likely to be caused by pathogens susceptible to antimicrobial therapy. ${ }^{1}$ In comparison, late-onset HAP (occurring 5 days or more within current hospitalization) is more likely due to multidrug-resistant (MDR) pathogens. ${ }^{1}$ Late-onset HAP and VAP caused by MDR pathogens is associated with increased morbidity and mortality. ${ }^{1}$

Consequences of HAP are further compounded by the limited antimicrobial treatment options available to combat this growing health care problem. Currently, vancomycin and linezolid are the only recommended therapies for HAP caused by MRSA. ${ }^{1}$ Even more concerning is that recent evidence suggests that MRSA isolates with a minimum inhibitory concentration (MIC) of $2 \mu \mathrm{g} / \mathrm{mL}$ do not respond as well to vancomycin as those isolates with an $\mathrm{MIC}$ of $\leq 0.5 \mu \mathrm{g} / \mathrm{mL}{ }^{7}$ The serious consequences of HAP coupled with limited treatment options emphasize the need for more available antistaphylococcal agents for the treatment of this infection.

Table I Risk factors for hospital-acquired pneumonia ${ }^{1,5,6}$

\begin{tabular}{ll}
\hline Modifiable risk factors & Nonmodifiable risk factors \\
\hline - Intubation & - Extremes of age \\
- Duration of mechanical & - Chronic lung disease (especially \\
ventilation & bronchitis, chronic obstructive \\
- Aspiration & pulmonary disease and asthma) \\
- Body position (supine & - Abdominal or thoracic surgery \\
versus semi-recumbent) & - Intubation \\
- Enteral feeding & - Duration of mechanical \\
- Modulation of colonization & ventilation \\
(eg, decontamination) & - Immunosuppression \\
- Stress ulcer prophylaxis & - Prior antimicrobial use \\
- Transfusions & \\
\hline
\end{tabular}

This article provides an overview of telavancin, including its clinical efficacy and safety profile, and evaluates its potential role in the treatment of nosocomial pneumonia caused by MRSA.

\section{Overview of telavancin}

Telavancin (Vibativ ${ }^{\mathrm{TM}}$; Theravance, San Francisco, CA, USA) is a bactericidal, lipoglycopeptide antibiotic that is structurally related to vancomycin. ${ }^{8}$ It is an intravenous, semi-synthetic product with concentration-dependent, antimicrobial activity against Gram-positive aerobic and anaerobic bacteria. ${ }^{9}$ Telavancin's mechanism of action is twofold. ${ }^{10}$ It inhibits bacterial cell wall synthesis by interfering with the polymerization and cross-linking of peptidoglycan. Also, telavancin binds to the bacterial membrane and disrupts membrane barrier function. Telavancin has been associated with a tenfold greater peptidoglycan synthesis inhibitory activity in intact MRSA cells compared to vancomycin. ${ }^{11}$ In initial clinical trials and surveillance studies, telavancin demonstrated in vitro activity against organisms that commonly cause skin and skin-structure infections and Gram-positive bacteria that cause pneumonia, including Staphylococcus spp., Streptococcus spp., and some Enterococcus spp. ${ }^{12}$ Telavancin does not exhibit appreciable activity against the most common type of vancomycinresistant enterococci, Van A-producing strains. ${ }^{12-14}$ However, telavancin may have some activity against Van B- and Van C-producing strains, depending on the free drug concentrations achieved. ${ }^{12-14}$ Currently telavancin is approved by the US Food and Drug Administration (FDA) for the treatment of adult patients with complicated skin and skin-structure infections (cSSSI) caused by susceptible Gram-positive bacteria. A summary of telavancin dosing recommendations, considerations in special populations, and potential interactions is provided in Table 2 .

\section{Activity against Staphylococcus aureus}

Telavancin demonstrated in vitro bactericidal activity against methicillin-susceptible S. aureus (MSSA) with an $\mathrm{MIC}_{90}$ (minimum inhibitory concentration required to inhibit the growth of $90 \%$ of organisms) of $0.12-1 \mu \mathrm{g} / \mathrm{mL}$ compared to $1-2 \mu \mathrm{g} / \mathrm{mL}$ for vancomycin. ${ }^{10,15}$ Similar in vitro activity against MRSA was also observed with an $\mathrm{MIC}_{90}$ of $0.25-1 \mu \mathrm{g} / \mathrm{mL}$ compared to $1-2 \mu \mathrm{g} / \mathrm{mL}$ for vancomycin. ${ }^{9,10,16}$ Currently, the Clinical and Laboratory Standards Institute (CLSI) has not published susceptibility breakpoints for telavancin. However, the FDA-approved MIC breakpoint for $S$. aureus, including MRSA, is $\leq 1 \mu \mathrm{g} / \mathrm{mL}$ using the broth dilution method. Although intermediate and resistant 
Table 2 Telavancin dosing, use in special populations, and interactions ${ }^{17,19,30,41-46}$

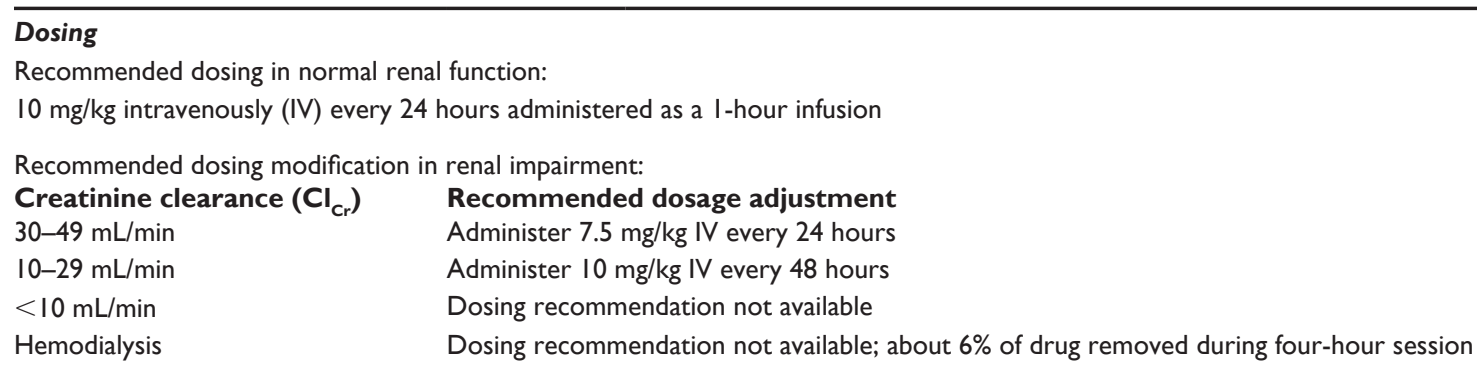

Special populations

Sex and age: No clinical impact on the pharmacokinetic disposition of telavancin

Pregnancy: FDA pregnancy category $\mathrm{C}$

Black box warning for potential risk of abnormal fetal development (ie, reports of increased rates of digit and limb malformations in animal offspring) Prescribers encouraged to register pregnant women receiving telavancin or women may enroll themselves into a pregnancy exposure registry created by Theravance

\section{Interactions}

Drug-drug Interactions: No clinically significant interactions have been reported with the concomitant use of telavancin and other drugs

Drug-laboratory test interactions:

Coagulation tests affected

- INR (International Normalized Ratio)

- PT (Prothrombin time)

Coagulation tests not affected

- PPTT (Prothrombin time)

- Fibrinogen level

- Thrombin time

- Heparin level

- ACT (Activated Clotting time)

- D-dimer

breakpoints have not been established, the manufacturer recommends that isolates yielding results other than susceptible be subjected to additional testing. ${ }^{17}$

\section{Pharmacokinetics}

In healthy young adults, telavancin demonstrated linear pharmacokinetics following the IV administration of single doses ranging from 5 to $12.5 \mathrm{mg} / \mathrm{kg}$ and multiple doses ranging from 7.5 to $15 \mathrm{mg} / \mathrm{kg}$ once daily for up to 7 days. ${ }^{18}$ Steadystate concentrations were achieved by the third daily dose. At 24 hours post-infusion, serum concentrations from subjects given telavancin exceeded the $\mathrm{MIC}_{90}$ for MRSA and penicillinresistant Streptococcus pneumoniae strains, suggesting that telavancin is an effective once-daily antibacterial agent.

Telavancin has a small volume of distribution $(0.115 \mathrm{~L} / \mathrm{kg})$, with approximately 90 to $95 \%$ of the drug being bound to albumin. In pharmacokinetic studies, its elimination half-life $\left(t_{1 / 2}\right)$ was 7.5 hours in healthy adults who received a single dose and 9.11 hours in adults who received multiple doses, respectively. ${ }^{11,19}$ Telavancin primarily undergoes renal elimination and $65 \%$ to $72 \%$ of the drug is excreted unchanged after several doses. ${ }^{18}$

Though controversial, drug concentrations in epithelial lining fluid (ELF) have been used to evaluate drug penetration into the pulmonary tissues..$^{20}$ The intrapulmonary distribution of telavancin ( $10 \mathrm{mg} / \mathrm{kg}$ IV every 24 hours) was evaluated in 20 healthy individuals. ${ }^{21}$ Throughout the dosing interval, telavancin achieved concentrations up to eightfold and 85-fold in ELF and alveolar macrophages, respectively, above the $\mathrm{MIC}_{90}$ for MRSA $(0.5 \mu \mathrm{g} / \mathrm{mL})$. It was also noted that pulmonary surfactant did not affect the in vitro antibacterial activity of telavancin. Using these 20 subjects, Monte Carlo simulation and population pharmacokinetic modeling were performed to evaluate telavancin's penetration into ELF. Investigators reported a median ELF area under the curve (AUC) that was approximately $75 \%$ of the free plasma AUC. ${ }^{22}$ In comparison, vancomycin concentrations in lung tissue ranges from $5 \%$ to $41 \%$ of serum concentrations. ${ }^{23-25}$ Epithelial lining fluid penetration in critically injured patients was highly variable, with an overall serum to ELF ratio of 6:1. ${ }^{26}$ These observations suggest that penetration of vancomycin into pulmonary tissue and ELF is poor. ${ }^{25,26}$

\section{Pharmacodynamics}

Against $S$. aureus, telavancin exhibits concentrationdependent bactericidal activity with a post-antibiotic effect of 1 to 4 hours. ${ }^{12,27,28}$ The AUC/MIC ratio has been identified as the pharmacodynamic marker correlating to the drug's efficacy against $S$. aureus. ${ }^{21}$ An in vitro study demonstrated that the maximal killing against $S$. aureus was achieved at an AUC/MIC ratio of $404 .{ }^{29}$ Alternatively, the lowest AUC/MIC ratio yielding no bacterial regrowth was 50 . In order to 
minimize the emergence of resistance, it has been suggested that the telavancin AUC/MIC ratio remain above 50. ${ }^{30}$

\section{Animal models of pneumonia}

The efficacy of telavancin and vancomycin against MRSA strains with vancomycin MICs $\geq 1 \mu \mathrm{g} / \mathrm{mL}$ was compared in a neutropenic murine model of pneumonia. ${ }^{31}$ Mice were administered antibiotic doses designed to simulate the area under the concentration-time curve (AUC) observed in humans given telavancin $10 \mathrm{mg} / \mathrm{kg}$ IV every 24 hours or vancomycin $1 \mathrm{~g}$ IV every 12 hours. Thirteen clinical MRSA isolates (one vancomycin-susceptible, two vancomycin-heteroresistant, and four vancomycin-intermediate) were tested after 24 hours and seven isolates (one vancomycin-heteroresistant, and four vancomycin-intermediate) were tested after 48 hours of exposure to the drug. Efficacy was expressed as the 24 or 48 hour change in lung bacterial density from pretreatment counts. During both time points, similar colony-forming unit (CFU) reductions were demonstrated for telavancin and vancomycin against MRSA isolates with vancomycin MICs of $2 \mu \mathrm{g} / \mathrm{mL}$ or less. Both telavancin and vancomycin demonstrated similar efficacy following 24 and 48 hours of exposure against the vancomycin-heteroresistant strains tested. Against vancomycin-intermediate isolates, telavancin reduced bacterial burdens more than vancomycin for one of four isolates after 24 hours and for three of four isolates after 48 hours.

Another study compared telavancin to vancomycin and linezolid in a neutropenic murine model of MRSA pneumonia. ${ }^{32}$ The MICs of telavancin, vancomycin, and linezolid against MRSA were $0.5,1$, and $1 \mu \mathrm{g} / \mathrm{mL}$, respectively. Mice were administered antibiotic doses that closely approximated human exposures at doses of 5 and $10 \mathrm{mg} / \mathrm{kg} \mathrm{IV}$ for telavancin, $1 \mathrm{~g}$ IV every 12 hours for vancomycin, and $600 \mathrm{mg}$ IV every 12 hours for linezolid. ${ }^{32,33}$ Mice treated with telavancin demonstrated a significantly greater reduction in lung bacterial titers at 48 hours compared to the mice treated with vancomycin or linezolid.

\section{Clinical studies: ATTAIN I and ATTAIN 2}

The efficacy of telavancin for the treatment of HAP, HCAP, and VAP due to Gram-positive pathogens, specifically MRSA, was evaluated in two identical randomized, multinational, noninferiority trials. ${ }^{34}$ Eligible patients were adult nonpregnant females or males who showed clinical signs and symptoms consistent with nosocomial pneumonia. Patients were required to have specific signs and symptoms of pneumonia, radiographic findings consistent with pneumonia, and a sufficient respiratory specimen for microbiologic evaluation. Exclusion criteria included prior receipt of potentially effective antibiotic therapy for Gram-positive pneumonia, Gram stain or culture revealing only Gram-negative bacteria, presence of certain pulmonary diseases (lung cancer, active tuberculosis, cystic fibrosis, or granulomatous disease), uncompensated heart failure, absolute neutrophil count $<500$ cells $/ \mathrm{mm}^{3}$, and baseline QTc interval greater than 500 milliseconds.

Patients were randomized in a $1: 1$ fashion to receive either telavancin, $10 \mathrm{mg} / \mathrm{kg}$ IV every 24 hours, or vancomycin, $1 \mathrm{~g}$ IV every 12 hours, for 7 to 21 days. Telavancin dosage adjustments were permitted in patients with creatinine clearance $\left(\mathrm{Cl}_{\mathrm{Cr}}\right)$ of $50 \mathrm{~mL} / \mathrm{min}$ or less. Vancomycin regimens were monitored and adjusted according to institutional policy at each site. The primary end point was clinical response (cure or failure) at follow-up/test-of-cure visit in the all-treated (AT) and clinically-evaluable (CE) populations, with a prespecified noninferiority margin of $20 \%$. The AT population included patients who were randomized and received at least one dose of the study drug. The CE population included patients in the AT population who were protocol-adherent or who died from the HAP episode after study day 3 . Results of the two identical studies were pooled for analysis. Secondary outcomes such as clinical response rate by identified pathogen, mortality, and safety parameters were also evaluated.

Of the 1532 patients randomized, 1,503 received at least one dose of the study drug (telavancin, $\mathrm{n}=749$; vancomycin, $\mathrm{n}=754$; AT population). A total of 654 patients were included in the $\mathrm{CE}$ population (telavancin, $\mathrm{n}=312$; vancomycin, $n=342$ ). Patients in both groups were comparable in terms of baseline and demographic variables. More than half of the patients in both groups were aged 65 years or older and more than half of the patients were in the intensive care unit at baseline. About $25 \%$ of patients in both groups had APACHE II scores greater than 20. Common comorbidities included diabetes, chronic obstructive pulmonary disease, and renal failure (acute and/or chronic). About one-third of patients in both groups had a $\mathrm{Cl}_{\mathrm{Cr}}$ of $50 \mathrm{~mL} / \mathrm{min}$ or less. More than half of the patients in both groups received previous antibiotics for greater than 24 hours.

Monomicrobial pneumonia caused by $S$. aureus was present in a total of 298 patients, and MRSA was the major $(60 \%)$ pathogen isolated from the respiratory tract. Mixed (Gram-positive and Gram-negative) infections were present in $27 \%$ of patients. Bacteremia was diagnosed in approximately six percent of patients. The $\mathrm{MIC}_{90}$ for 
both MSSA and MRSA was $0.5 \mu \mathrm{g} / \mathrm{mL}$ for telavancin and $1 \mu \mathrm{g} / \mathrm{mL}$ for vancomycin. In those patients for which vancomycin serum concentration monitoring was performed ( $\mathrm{n}=226$ ), the mean trough was $\geq 5 \mu \mathrm{g} / \mathrm{mL}$ in $94 \%$ of patients and $\geq 10 \mu \mathrm{g} / \mathrm{mL}$ in $66 \%$ of patients.

In the AT population, cure rates were 58.9\% for telavancin and $59.5 \%$ for vancomycin $(95 \%$ confidence interval [CI]: $-5.6 \%$ to $4.3 \%$ ). In the $\mathrm{CE}$ population, cure rates were $82.4 \%$ for telavancin and $80.7 \%$ for vancomycin (95\% CI: $-4.3 \%$ to $7.7 \%$ ). Based on these results, telavancin's noninferiority to vancomycin was demonstrated.

In secondary analysis, clinical response in patients with pneumonia due to MRSA with or without other pathogens was also similar between the two treatment groups. However, treatment with telavancin was associated with higher cure rates in patients with MSSA (87.9\% versus $75 \%$; $95 \%$ CI: $-4.2 \%$ to $28.8 \%$ ) and monomicrobial MRSA infection ( $81.8 \%$ versus $74.1 \%$; $95 \%$ CI: $-3.5 \%$ to $19.3 \%$ ). Similarly, higher cure rates with telavancin were observed among patients infected with $S$. aureus with a vancomycin $\mathrm{MIC} \geq 1 \mu \mathrm{g} / \mathrm{mL}(87.1 \%$ versus $74.3 \% ; P=0.03)$. Cure rates were lower for telavancin in patients with mixed infections $(66.2 \%$ versus $79.4 \%$; $95 \% \mathrm{CI}$ : $-26.9 \%$ to $3.2 \%)$. However, cure rates were similar between treatment groups in patients with mixed infections who received adequate Gram-negative antimicrobial coverage (63.2\% versus $66.7 \%$; $95 \% \mathrm{CI}:-28.9 \%$ to $25.7 \%$ ). There were no significant differences in mortality between treatment groups (20\% for telavancin versus $18.6 \%$ for vancomycin, $95 \% \mathrm{CI}:-2.6 \%$ to $5.3 \%$ ).

\section{Safety}

More patients experienced serious adverse events that lead to drug discontinuation in the telavancin group compared to the vancomycin group ( $8 \%$ versus $5 \%$ ). The most common adverse effects reported were nausea, anemia, hypokalemia, diarrhea, and constipation. Clinically significant increases in serum creatinine were more frequent among the telavancin group compared to the vancomycin group (16\% versus 10\%). Drug-related increases in serum creatinine associated with telavancin were mild and reversible after drug discontinuation. Prolongation of the QTc interval > 60 milliseconds occurred in $8 \%$ of telavancin-treated patients and $7 \%$ of vancomycin-treated patients. A maximum QTc interval $>500$ milliseconds occurred in $2 \%$ of patients in each group, and no patients experienced arrhythmias attributable to a prolonged QTc interval.

\section{Limitations of the ATTAIN studies}

Due to variations by country in the standard of care for pneumonia diagnosis, a limited number of patients in the ATTAIN studies underwent semi-invasive procedures such as bronchoalveolar lavage (BAL). Therefore, determination of the exact microbial pathogen in these studies may be less reliable. Respiratory tract samples (invasive or noninvasive) were obtained in approximately $30 \%$ of patients overall.

Authors reported that the majority of patients achieved "adequate" mean vancomycin serum concentrations (5-15 $\mu \mathrm{g} / \mathrm{mL})$. However, for the treatment of health careassociated pneumonia (including HAP and VAP), the recommended trough goal is $15-20 \mu \mathrm{g} / \mathrm{mL}$. ${ }^{1,35,36}$ This trough goal should be considered for invasive infections such as HAP, and may increase the likelihood of achieving the target AUC/MIC ratio of 400 (when the MRSA MIC is $<2 \mu \mathrm{g} / \mathrm{mL}) .{ }^{35}$ Furthermore, since vancomycin troughs $<10 \mu \mathrm{g} / \mathrm{mL}$ have been associated with the emergence of resistance, this should generally be avoided. ${ }^{35,37}$ In the ATTAIN studies, telavancin was likely compared to suboptimal vancomycin therapy suboptimal vancomycin therapy as evidenced by only evidenced by only $66 \%$ of patients with a trough $\geq 10 \mu \mathrm{g} / \mathrm{mL}$. A comparison of telavancin to dose-optimized vancomycin could serve to further validate the findings of the ATTAIN studies.

\section{Clinical utility of telavancin}

Telavancin provides advantages in the treatment of nosocomial pneumonia due to MRSA compared to other antistaphylococcal agents. It exhibits rapid bactericidal activity, whereas vancomycin demonstrates relatively slow bactericidal activity. Additional advantages over vancomycin include once-daily dosing, the lack of serum concentration monitoring, and a low incidence of infusion-related reactions. Telavancin lacks clinically relevant drug interactions, which may be an advantage over linezolid. However, telavancin is available only as an intravenous preparation. Also, telavancin has demonstrated in vitro and clinical efficacy in the treatment of pneumonia, an advantage over daptomycin.

Higher vancomycin MICs in MRSA are correlated with a greater likelihood of treatment failure..$^{23,35,36}$ The fact that MICs have continued to increase in $S$. aureus strains highlights the need for additional effective antibacterial agents. ${ }^{34}$ Though a secondary outcome in the ATTAIN studies, higher cure rates with telavancin were observed in patients 
infected with MRSA with a vancomycin MIC $\geq 1 \mu \mathrm{g} / \mathrm{mL}$. This preliminary evidence suggests that telavancin may play a role in treating this subset of patients. However, this requires further study.

Results of two retrospective analyses and one prospective study suggest that linezolid is superior to vancomycin for the treatment of MRSA nosocomial pneumonia. ${ }^{38,39}$ In light of these findings, subsequent studies should evaluate the efficacy of telavancin compared to linezolid for MRSA nosocomial pneumonia. Furthermore, comparing telavancin, linezolid, and dose-optimized vancomycin simultaneously may provide additional insight into the agent of choice for this indication.

Though there are benefits to telavancin use, additional research is necessary in some areas. At the present time evidence does not support the use of telavancin in patients with severe renal insufficiency, therefore limiting its use to patients with a $\mathrm{Cl}_{\mathrm{Cr}}>10 \mathrm{~mL} / \mathrm{min}$. The acquisition cost of telavancin may limit its use in health care facilities. Although pharmacoeconomic analysis is not yet available, the reported average wholesale price of telavancin is approximately $\$ 150$ per day compared to $\$ 20$ per day for vancomycin. ${ }^{40}$ Additional clinical studies are needed to evaluate the efficacy of telavancin for the treatment of other serious Gram-positive infections, such as bacteremia and endocarditis, as well.

\section{Conclusion}

In the setting of limited options for the treatment of nosocomial pneumonia due to MRSA, telavancin represents an effective alternative to standard therapy. Telavancin was associated with higher cure rates among MRSA strains with a vancomycin $\mathrm{MIC} \geq 1 \mu \mathrm{g} / \mathrm{mL}$, providing a potential role to be further explored. Overall, telavancin is well tolerated, with the most commonly experienced side effects being gastrointestinal intolerance and mild, reversible elevations in serum creatinine.

\section{Disclosure}

The authors report no conflicts of interest in this work.

\section{References}

1. American Thoracic Society and the Infectious Diseases Society America. Guidelines for the management of adults with hospital-acquired, ventilator-associated, and healthcare-associated pneumonia. Am J Respir Crit Care Med. 2005;171(4):388-416.

2. Kollef MH, Shorr A, Tabak YP, et al. Epidemiology and outcomes of healthcare-associated pneumonia: results from a large US database of culture-positive pneumonia. Chest. 2005;128(6):3854-3862.

3. Chastre J, Fagon JY. Ventilator-associated pneumonia. Am J Respir Crit Care Med. 2002;165(7):867-903.
4. Tablan OC, Anderson LJ, Besser R, et al. Healthcare Infection Control Practices Advisory Committee, Centers for Disease Control and Prevention. Guidelines for preventing healthcare-associated pneumonia, 2003: recommendations of the CDC and the Healthcare Infection Control Practices Advisory Commitee. MMWR. 2004;53(RR-3):1-36.

5. Kollef MH. The prevention of ventilator-associated pneumonia. NEngl J Med. 1999;340(8):627-634.

6. Fridkin SK, Welbel SF, Weinstein RA. Magnitude and prevention of nosocomial infections in the intensive care unit. Infect Dis Clin North Am. 1997;11(2):479-496.

7. Sakoulas G, Moise-Broder PA, Schentag J, Forrest A, Moellering RC Jr, Eliopoulos GM. Relationship of MIC and bactericidal activity to efficacy of vancomycin for treatment of methicillin-resistant Staphylococcus aureus bacteremia. J Clin Microbiol. 2004;42(6):2398-2402.

8. Leadbetter MR, Adams SM, Bazzini B, et al. Hydrophobic vancomycin derivatives with improved ADME properties: Discovery of telavancin (TD-6424). J Antibiot. 2004;57(5):326-336.

9. King A, Phillips I, Kaniga K. Comparative in vitro activity of telavancin (TD-6424), a rapidly bactericidal, concentration-dependent antiinfective with multiple mechanisms of action against Gram-positive bacteria. J Antimicrob Chemother. 2004;53(5):797-803.

10. Pace JL, Krause K, Johnston D, et al. In vitro activity of TD-6424 against Staphylococcus aureus. Antimicrob Agents Chemother. 2003; 47(11):3602-3604.

11. Higgins DL, Chang R, Debabov DV, et al. Telavancin, a multifunctional lipoglycopeptide, disrupts both cell wall synthesis and cell membrane integrity in methicillin-resistant Staphylococcus aureus. Antimicrob Agents Chemother. 2005;49(3):1127-1134.

12. Draghi DC, Benton BM, Krause KM, Thornsberry C, Pillar C, Sahm DF. Comparative surveillance study of telavancin activity against recently collected gram-positive clinical isolates from across the United States. Antimicrob Agents Chemother. 2008;52(7):2383-2388.

13. Krause KM, Renelli M, Difuntorum S, Wu TX, Debabov DV, Benton BM. In vitro activity of telavancin against resistant gram-positive bacteria. Antimicrob Agents Chemother. 2008;52(7):2647-2652.

14. Saravolatz LD, Stein GE, Johnson LB. Telavancin: a novel lipoglycopeptide. Clin Infect Dis. 2009;49(12):1908-1914.

15. Jansen WTA, Verel A, Verhoef J, Milatovic D. In vitro activity of telavancin against gram-positive clinical isolates recently obtained in Europe. Antimicrob Agents Chemother. 2007;51(9):3420-3424.

16. Hatano K, Matsuzaki K, Sato Y, Kobayashi I, Yamaguchi K. In vitro antimicrobial activity of telavancin against methicillin-resistant Staphylococcus aureus clinical isolates from Japan (2006). J Antibiot. 2007;60(11):709-712.

17. Theravance, Inc. Vibativ (Package Insert). South San Francisco, CA: Theravance, Inc; 2009.

18. Shaw JP, Seroogy J, Kaniga K, Higgins DL, Kitt M, Barriere S. Pharmacokinetics, serum inhibitory and bactericidal activity, and safety of telavancin in healthy subjects. Antimicrob Agents Chemother. 2005;49(1):195-201.

19. Wong SL, Barriere SL, Kitt MM, Goldberg MR. Multiple-dose pharmacokinetics of intravenous telavancin in healthy male and female subjects. J Antimicrob Chemother. 2008;62(4):780-783.

20. Ambrose PG, Bhavnani SM, Ellis-Grosse EJ, Drusano GL. Pharmacokinetic-pharmacodynamic considerations in the design of hospital-acquired or ventilator-associated bacterial pneumonia studies: look before you leap! Clin Infect Dis. 2010;51(Suppl 1):S103-S110.

21. Gotfried MH, Shaw JP, Benton BM, et al. Intrapulmonary distribution of intravenous telavancin in healthy subjects and effect of pulmonary surfactant on in vitro activities of telavancin and other antibiotics. Antimicrob Agents Chemother. 2008;52(1):92-97.

22. Lodise TP, Gotfried M, Barriere S, Drusano GL. Telavancin penetration into human epithelial lining fluid determined by population pharmacokinetic modeling and Monte Carlo simulation. Antimicrob Agents Chemother. 2008;52(7):2300-2304.

23. Stevens DL. The role of vancomycin in the treatment paradigm. Clin Infect Dis. 2006;42(Suppl 1):S51-S57. 
24. Rybak MJ. The pharmacokinetic and pharmacodynamic properties of vancomycin. Clin Infect Dis. 2006;42(Suppl 1):S35-S39.

25. Cruciani M, Gatti G, Lazzarini L, et al. Penetration of vancomycin into human lung tissue. J Antimicrob Chemother. 1996;38(5):865-869.

26. Lamer C, de Beco V, Soler P, et al. Analysis of vancomycin entry into pulmonary lining fluid by bronchoalveolar lavage in critically ill patients. Antimicrob Agents Chemother. 1993;37(2):281-286.

27. Hegde SS, Reyes N, Wiens T, et al. Pharmacodynamics of telavancin (TD-6424), a novel bactericidal agent, against gram-positive bacteria. Antimicrob Agents Chemother. 2004;48(8):3043-3050.

28. Pankuch GA, Appelbaum PC. Postantibiotic effects of telavancin against 16 Gram-positive organisms. Antimicrob Agents Chemother. 2009;53(3):1275-1277.

29. Odenholt I, Lowdin E, Cars O. Pharmacodynamic effects of telavancin against methicillin-resistant and methicillin-susceptible Staphylococcus aureus strains in the presence of human albumin or serum and in an in vitro kinetic model. Antimicrob Agents Chemother. 2007;51(9):3311-3316.

30. MacGowan AP, Noel AR, Tomaselli S, Elliott HC, Bowker KE. Pharmacodynamics of telavancin studied in an in vitro pharmacokinetic model of infection. Antimicrob Agents Chemother. 2011;55(2):867-873.

31. Crandon JL, Kuti JL, Nicolau DP. Comparative efficacies of human simulated exposures of telavancin and vancomycin against methicillin-resistant Staphylococcus aureus with a range of vancomycin MICs in a murine pneumonia model. Antimicrob Agents Chemother. 2010;54(12):5115-5119.

32. Reyes N, Skinner R, Kaniga K, et al. Efficacy of telavancin (TD-6424), a rapidly bactericidal lipoglycopeptide with multiple mechanisms of action, in a murine model of pneumonia induced by methicillinresistant Staphylococcus aureus. Antimicrob Agents Chemother. 2005;49(10):4344-4346.

33. Slatter JG, Adams LA, Bush EC, et al. Pharmacokinetics, toxicokinetics, distribution, metabolism and excretion of linezolid in mouse, rat and dog. Xenobiotica. 2002;32(10):907-924.

34. Rubinstein E, Lalani T, Corey GR, et al. Telavancin versus vancomycin for hospital-acquired pneumonia due to gram-positive pathogens. Clin Infect Dis. 2011;52(1):31-40.

35. Rybak M, Lomaestro B, Rotschafer JC, et al. Therapeutic monitoring of vancomycin in adult patients: a consensus review of the American Society of Health-System Pharmacists, the Infectious Diseases Society of America, and the Society of Infectious Diseases Pharmacists. Am J Health Syst Pharm. 2009;66(1):82-98.
36. Liu C, Bayer A, Cosgrove SE, et al. Clinical practice guidelines by the infectious diseases society of america for the treatment of methicillinresistant Staphylococcus aureus infections in adults and children: executive summary. Clin Infect Dis. 2011;52(3):285-292.

37. Howden BP, Ward PB, Charles PG, et al. Treatment outcomes for serious infections caused by methicillin-resistant Staphylococcus aureus with reduced vancomycin susceptibility. Clin Infect Dis. 2004;38(4):521-528.

38. Wunderink RG, Rello J, Cammarata SK, Croos-Dabrera RV, Kollef MH. Linezolid vs vancomycin: analysis of two double-blind studies of patients with methicillin-resistant Staphylococcus aureus nosocomial pneumonia. Chest. 2003;124(5):1789-1797.

39. Wunderink RG, Niederman MS, Kollef MH, et al. Linezolid in methicillin-resistant Staphylococcus aureus nosocomial pneumonia: a randomized, controlled study. Clin Infect Dis. 2012;54(5):621-629.

40. Rubinstein E, Corey GR, Stryjewski ME, Kanafani ZA. Telavancin for the treatment of serious gram-positive infections, including hospital acquired pneumonia. Expert Opin Pharmacother. 2011;12(17): 2737-2750.

41. Duchin K, Shaw J, Spencer E, Seroogy J, Barriere S, Wilbraham D. Single dose pharmacokinetics of telavancin in subjects with renal dysfunction. Clin Microbiol Infect. 2004;10(Suppl 3):P1028.

42. Duchin K, Shaw J, Seroogy J, Barriere S, Novack R. Effect of hemodialysis on single dose pharmacokinetics of telavancin. Clin Microbiol Infect. 2005;11(Suppl 2):P897.

43. Goldberg MR, Wong SL, Shaw JP, Kitt MM, Barriere SL. Single-dose pharmacokinetics and tolerability of telavancin in elderly men and women. Pharmacotherapy. 2010;30(8):806-811.

44. Wong SL, Sorgel F, Kinzig M, Goldberg MR, Kitt MM, Barriere SL. Lack of pharmacokinetic drug interactions following concomitant administration of telavancin with aztreonam or piperacillin/tazobactam in healthy participants. J Clin Pharmacol. 2009;49(7):816-823.

45. Wong SL, Goldberg MR, Ballow CH, Kitt MM, Barriere SL. Effect of telavancin on the pharmacokinetics of the cytochrome $\mathrm{P} 450$ 3A probe substrate midazolam: a randomized, double-blind, crossover study in healthy subjects. Pharmacotherapy. 2010;30(2):136-143.

46. Gosselin R, Dager W, Roberts A, et al. Effect of telavancin (Vibativ) on routine coagulation test results. Am J Clin Pathol. 2011;136(6): $848-854$.
Therapeutics and Clinical Risk Management

\section{Publish your work in this journal}

Therapeutics and Clinical Risk Management is an international, peerreviewed journal of clinical therapeutics and risk management, focusing on concise rapid reporting of clinical studies in all therapeutic areas, outcomes, safety, and programs for the effective, safe, and sustained use of medicines. This journal is indexed on PubMed Central, CAS,

\section{Dovepress}

EMBase, Scopus and the Elsevier Bibliographic databases. The manuscript management system is completely online and includes a very quick and fair peer-review system, which is all easy to use. Visit http://www.dovepress.com/testimonials.php to read real quotes from published authors. 